\title{
Kinematic Frenkel gap biexciton in one-dimensional structures
}

\author{
V.M. Agranovich*, O.A. Dubovsky, A.M. Kamchatnov \\ Institute of Spectroscopy, Russian Academy of Sciences, Troitsk, Moscow Reg. 142092, Russia
}

\begin{abstract}
We consider the role of kinematical exciton-exciton interaction in two-particle spectra of Frenkel excitons induced by their paulion nature. We demonstrate that, even if the dynamical exciton-exciton interaction is absent, in the 1D crystals with several molecules in the unit cell, the bound states of two excitons may be formed within a gap between the bands of two-particle states. Numerical analysis of dispersion relation for such gap biexcitons shows that these type of biexcitons have band's width small compared with the band's width of free two exciton states. We can expect that these states can give essential contribution into the resonant part of the nonlinear optical polarizability and two-photon absorption of 1D molecular aggregates. (C) 2001 Elsevier Science B.V. All rights reserved.
\end{abstract}

Keywords: Excitation spectra calculations; Many-body and quasiparticle theories

\section{Introduction}

The problem of electronic biexcitons (excitonic molecules) in molecular crystals was discussed repeatedly (see [1] and references therein). Such attention is motivated by the important role which, as in the case of semiconductors [2], Frenkel biexcitons could play in the processes of photoluminescence, hyper-Raman scattering, two-photon absorption and four-wave mixing. It is usually supposed that attraction between excitons is necessary for biexciton states to appear [3,4]. It is well known [5], however, that Frenkel excitons are the quasiparticles whose creation and annihilation operators obey the Pauli commutation relations: two paulion operators for excitons situated at molecules $n$ and $m$ behave like those for bosons when $n \neq m$, whereas the operators for the same molecule $(n=m)$ anticommute like those for fermions. This means that two Frenkel excitons cannot be located at one molecule, and hence the paulion statistic may be interpreted as strong repulsion of excitons, i.e. as their "kinematical" interaction. It has been predicted recently [6], that under certain conditions this kinematical repulsion is sufficient for formation of bound biexciton states. This effect is similar to formation of the localized state near vacancy [5]. In this case a localized exciton state exists if only there are several exciton bands in the spectrum as, for example, it takes place in vicinity of Davydov multiplet. Correspondingly, kinematical biexciton states

\footnotetext{
*Corresponding author. Tel.: +39-50-50-9066; fax: +39-50-56-3513. E-mail address: agran@sab.sns.it (V.M. Agranovich).
}

can exist only in crystals with similar structure of excitonic spectrum [6]. The crucial importance of this condition was illustrated by simple exactly solvable $1 \mathrm{D}$ model with symmetric structure, for which the biexciton state manifests itself as a resonant peak in the continuum spectrum of twoparticle exciton states. This paper is devoted to the theory of kinematic biexcitons for 1D crystals with asymmetric unit structure. More specifically, the theory will be applied to 1D crystals with two molecules in unit cell and parameters' values similar to those for anthracene. In such crystals the lowest energy excitonic spectrum consists of two excitonic bands, $\mu=1,2$ (Davydov splitting). Hence, the spectrum of two-exciton unbound states consists of three bands $(1,1),(1,2)$ and $(2,2)$. We show that for such a structure a new type of biexciton state, gap biexciton, appears.

\section{Theory}

For crystals with $\sigma$ molecules in unit cell, the Hamiltonian in site representation has the form

$H=\sum_{n, \alpha} E_{0} P_{\boldsymbol{n}, \alpha}^{\dagger} P_{\boldsymbol{n} \alpha}+\sum_{\boldsymbol{n}, \alpha \neq \boldsymbol{m}, \beta} V_{\boldsymbol{n} \boldsymbol{m}}^{\alpha \beta} P_{\boldsymbol{n}, \alpha}^{\dagger} P_{\boldsymbol{m} \beta}$,

where $E_{0}$ is the exciton energy in an individual molecule, $P_{n, \alpha}^{\dagger}$ and $P_{n \alpha \alpha}$ the creation and annihilation operators of exciton on the molecule $\alpha, \alpha=1, \ldots, \sigma$, in the site $\boldsymbol{n}$, obeying Pauli commutation relations, $V_{n m}^{\alpha \beta}$ is the energy of intermolecular interaction. The Schrödinger equation

$H|2\rangle=E|2\rangle$ 
for two-exciton state

$|2\rangle=\sum_{\boldsymbol{n}, \alpha \neq \boldsymbol{m}, \beta} \Psi_{\boldsymbol{n} \boldsymbol{m}}^{\alpha \beta} P_{\boldsymbol{n}, \alpha}^{\dagger} P_{\boldsymbol{m}, \beta}^{\dagger}|0\rangle$

leads to the following equations for the wave functions $\Psi_{n \boldsymbol{m}}^{\alpha \beta}$ (see Appendix in [6])

$\left(E-2 E_{0}\right) \Psi_{\boldsymbol{n} \boldsymbol{m}}^{\alpha \beta}=\sum_{\boldsymbol{l} \gamma}\left(V_{\boldsymbol{n} \boldsymbol{l}}^{\alpha \gamma} \Psi_{\boldsymbol{l m}}^{\gamma \beta}+V_{\boldsymbol{m} \boldsymbol{l}}^{\beta \gamma} \Psi_{\boldsymbol{n} l}^{\alpha \gamma}\right), \quad \Psi_{l l}^{\gamma \gamma}=0$.

Their solution with total wave vector $\boldsymbol{K}$ of biexciton has the form

$\Psi_{\boldsymbol{n} \boldsymbol{m}}^{\alpha \beta}(\boldsymbol{K})=\frac{1}{N} \sum_{\gamma \mu v, \boldsymbol{p}+\boldsymbol{q}=\boldsymbol{K}} \frac{R_{\gamma} u_{\mu, \boldsymbol{p}}^{\alpha} u_{v, \boldsymbol{q}}^{\beta} u_{\mu, \boldsymbol{p}}^{\gamma *} u_{v, \boldsymbol{q}}^{\gamma *} \mathrm{e}^{\mathrm{i}(\boldsymbol{p} \boldsymbol{n}+\boldsymbol{q m})}}{E-2 E_{0}-\epsilon_{\mu, \boldsymbol{p}}-\epsilon_{v, \boldsymbol{q}}}$,

where $\epsilon_{\mu, p}$ is a one-exciton energy in the band $\mu$ with wave vector $\boldsymbol{p}, u_{\mu, \boldsymbol{p}}^{\alpha}$ being the corresponding eigenfunction of the one-exciton problem. The coefficients $R_{\gamma}=R_{\gamma}(\boldsymbol{K})$ are determined as solutions of the system of linear equations,

$\sum_{\beta} T_{\alpha \beta}(E, \boldsymbol{K}) R_{\beta}(E, \boldsymbol{K})=0$,

where

$T_{\alpha \beta}(E, \boldsymbol{K})=\frac{1}{N} \sum_{\mu v, \boldsymbol{p}+\boldsymbol{q}=\boldsymbol{K}} \frac{u_{\mu, \boldsymbol{p}}^{\alpha} u_{v, \boldsymbol{q}}^{\alpha} u_{\mu, \boldsymbol{p}}^{\beta *} u_{v, \boldsymbol{q}}^{\beta *}}{E-2 E_{0}-\epsilon_{\mu, \boldsymbol{p}}-\epsilon_{v, \boldsymbol{q}}}$,

and the condition of solvability of the system (8),

$\operatorname{det}\left|T_{\alpha \beta}(E, \boldsymbol{K})\right|=0$,

defines the biexciton dispersion law. It is easy to verify that the above formulas give the solution of Eq. (4).

We consider 1D model with two molecules in unit cell $(\alpha=1,2)$ shown in Fig. 1 . Thus, the structure consists of two parallel chains of molecules with $\alpha=1$ and 2 ,

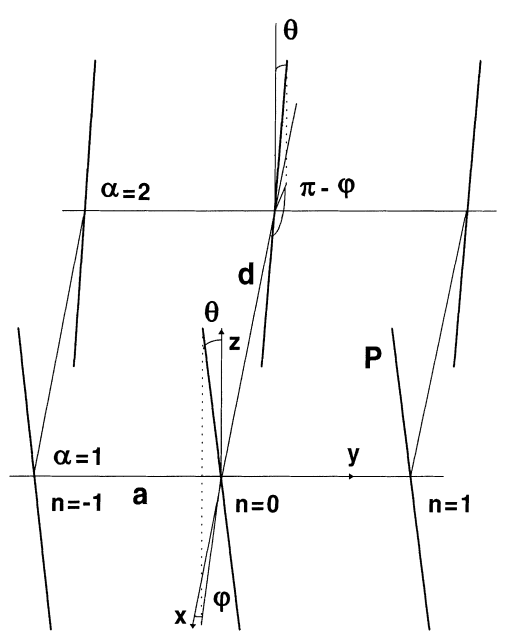

Fig. 1. Schematic sketch of 1D molecular crystal with two molecules in unit cell. Thick lines shows the directions of the transition dipole moments in molecules $\alpha=1$ and 2, the angles $\theta$ and $\phi$ are the corresponding spherical coordinates. correspondingly. The distance between two molecules along each chain (the lattice constant) equals to $a$, and the distance between chains equals to $d$, vectors $\boldsymbol{d}$ and $\boldsymbol{a}$ being perpendicular to each other. The directions of the dipole moments of optical transitions in the molecules 1 and 2 are determined, correspondingly, by the angles $\theta_{1}, \phi_{1}$ and $\theta_{2}, \phi_{2}$ in the spherical coordinates, where $(x, y)$ is the crystal's plane, $\boldsymbol{d}$ is directed along $x$-axis and $\boldsymbol{a}$ along $y$-axis. In contrast to earlier discussed symmetric case [6], now we choose values $\theta_{1}=\theta_{2}=\theta=0.1, \phi_{1}=\phi=0.1, \phi_{2}=\pi-\phi$, corresponding to the anthracene-like configuration. In our calculations, we take into account the dipole-dipole interaction of three nearest neighbors,

$V_{n \boldsymbol{n}}^{12}=\left[\left(\boldsymbol{p}_{1} \cdot \boldsymbol{p}_{2}\right) \boldsymbol{d}^{2}-3\left(\boldsymbol{p}_{1} \cdot \boldsymbol{d}\right)\left(\boldsymbol{p}_{2} \cdot \boldsymbol{d}\right)\right] d^{-5}$,

$V_{\boldsymbol{n}, \boldsymbol{n} \pm 1}=\left[p_{\alpha}^{2} a^{2}-3\left(\boldsymbol{p}_{\alpha} \cdot \boldsymbol{a}\right)^{2}\right] a^{-5}, \quad \alpha=1,2$,

$V_{n, \boldsymbol{n} \pm 1}^{\alpha \beta}=\left[\left(\boldsymbol{p}_{\alpha} \cdot \boldsymbol{p}_{\beta}\right)\left(\boldsymbol{d}^{2}+\boldsymbol{a}^{2}\right)-3\left(\boldsymbol{p}_{\alpha} \cdot(\boldsymbol{d}+\boldsymbol{a})\right)\left(\boldsymbol{p}_{\beta} \cdot(\boldsymbol{d}+\boldsymbol{a})\right)\right]$

$\times\left(d^{2}+a^{2}\right)^{-5 / 2}, \quad \alpha \neq \beta$.

To make gaps between bands much more than band's widths, we take $d / a=0.4$ which is close to the real value for the anthracene. All results are expressed in dimensionless variables with unit energy given by $\boldsymbol{V}=p^{2} / a^{3}, p=\left|\boldsymbol{p}_{\alpha}\right|$, and length unit equal to $a$. Diagonalization of matrix $\boldsymbol{V}$ yields the dispersion relation $\epsilon_{\mu, \boldsymbol{k}}$ for Davydov's doublet with negative effective mass at small values of the wave vector $\boldsymbol{k}$ :

$$
\begin{aligned}
\epsilon_{\mu, \boldsymbol{k}}= & (1 / 2)\left(V_{\boldsymbol{k}}^{11}+V_{\boldsymbol{k}}^{2}\right) \\
& \pm \sqrt{(1 / 4)\left(V_{\boldsymbol{k}}^{11}-V_{\boldsymbol{k}}^{2}\right)^{2}+\left(V_{00}^{12}+W_{\boldsymbol{k}}^{\prime}\right)^{2}+\left(W_{\boldsymbol{k}}^{\prime \prime}\right)^{2}}, \\
V_{\boldsymbol{k}}^{\alpha \beta}= & \sum_{m} V_{n m}^{\alpha \beta} \mathrm{e}^{\mathrm{i} \boldsymbol{k}(n-m)}, \quad V_{\boldsymbol{k}}^{12}=\left(V_{\boldsymbol{k}}^{21}\right)^{*} \equiv W_{\boldsymbol{k}}^{\prime}-\mathrm{i} W_{\boldsymbol{k}}^{\prime \prime} .
\end{aligned}
$$

The corresponding normalized wave functions have the form

$$
\begin{aligned}
& u_{1 k}^{1}=\sqrt{\frac{1}{2}\left(1+\frac{\delta_{k}}{D_{k}}\right)} \mathrm{e}^{(\mathrm{i} / 2) \xi_{k}}, \quad u_{1 k}^{2}=\sqrt{\frac{1}{2}\left(1-\frac{\delta_{k}}{D_{k}}\right)} \mathrm{e}^{(\mathrm{i} / 2) \xi_{k}}, \\
& u_{2 k}^{1}=\sqrt{\frac{1}{2}\left(1-\frac{\delta_{k}}{D_{k}}\right)} \mathrm{e}^{(\mathrm{i} / 2) \xi_{k}}, \quad u_{2 k}^{2}=-\sqrt{\frac{1}{2}\left(1+\frac{\delta_{k}}{D_{k}}\right)} \mathrm{e}^{(\mathrm{i} / 2) \xi_{k}},
\end{aligned}
$$

where

$$
\begin{aligned}
& \tan \xi_{k}=-\frac{W_{k}^{\prime \prime}}{V_{00}^{12}+W_{k}^{\prime}}, \quad \delta_{k} \equiv \frac{V_{k}^{11}-V_{k}^{22}}{2} \\
& D_{k} \equiv \sqrt{\delta_{k}^{2}+\left(V_{00}^{12}+W_{k}^{\prime}\right)^{2}+\left(W_{k}^{\prime \prime}\right)^{2}}
\end{aligned}
$$

These formulas were used in numerical calculations of the two-exciton spectrum of the crystal model with 25 unit cells and cyclic boundary conditions imposed on the wave functions. Control calculations have shown that increase of the number of unit cell does not change the results. The spectrum can be determined by finding the zeros in 


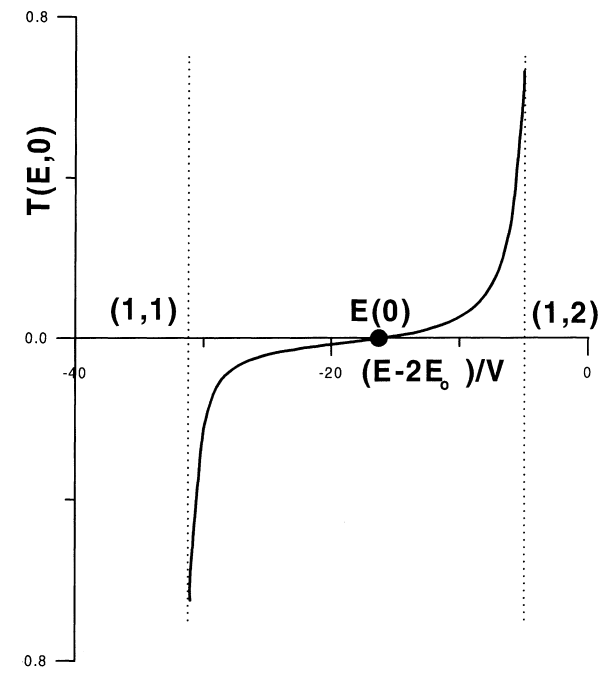

Fig. 2. The dependence of the determinant (18) on the energy $E$ at $\boldsymbol{K}=0$. The vertical dotted lines indicate the boundaries of the gap between the lowest energy bands of two particle states.

the dependence of the determinant

$T(E, \boldsymbol{K}) \equiv T_{11}(E, \boldsymbol{K}) T_{22}(E, \boldsymbol{K})-\left|T_{12}(E, \boldsymbol{K})\right|^{2}$

on the energy $E$ at fixed values of the wave vector $\boldsymbol{K}$. As one should expect, the function $T(E, \boldsymbol{K})$ vanishes at three values $\epsilon_{\mu, \boldsymbol{k}}+\epsilon_{v, \boldsymbol{K}-\boldsymbol{k}},(\mu, v)=(1,1),(1,2)$ and $(2,2)$, of unbound two-exciton states. These states correspond to scattering of excitons by each other. In addition, $T(E, \boldsymbol{K})$ has a zero at $E=E(\boldsymbol{K})$ within the gap between two bands $(1,1)$ and $(1,2)$. To illustrate this phenomenon, we have shown in Fig. 2 the dependence $T(E, 0)$ on $E$ at $\boldsymbol{K}=0$. The filled circle shows the position of the kinematical biexciton state with zero wave vector $\boldsymbol{K}$. Analogous calculations were made for a large enough set of values of $\boldsymbol{K}$ within the first Brilloin

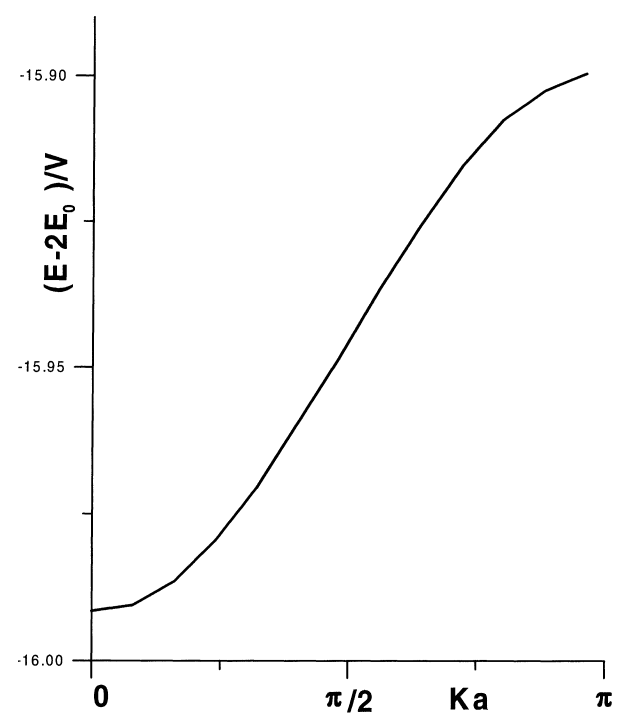

Fig. 3. The dependence of the kinematical gap biexciton energy on the wave vector.

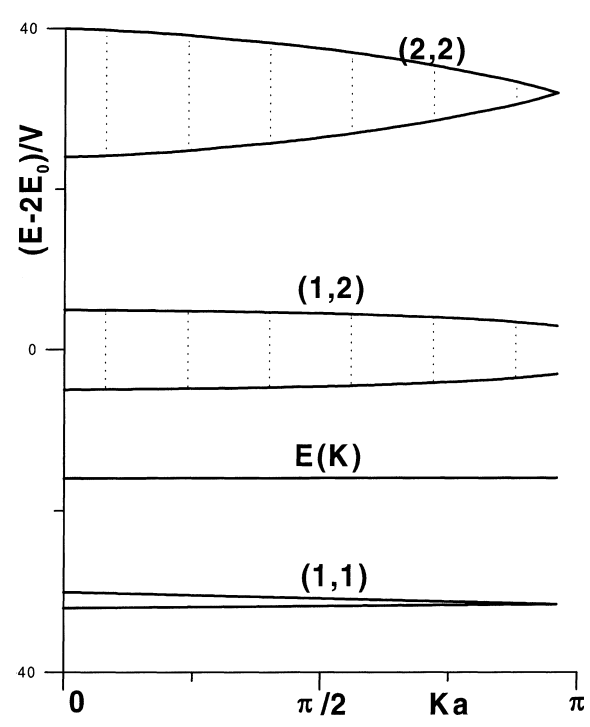

Fig. 4. Spectrum of excitonic states in 1D molecular crystal with two molecules in unit cell: bands of unbound two-particle excitonic states $(1,1),(1,2)$ and $(2,2)$ and gap biexciton.

zone, which allows us to reproduce the dispersion dependence $E(\boldsymbol{K})$. This biexciton dispersion dependence is shown in Fig. 3. Note that kinematical biexciton has positive effective mass at $\boldsymbol{K}=0$, whereas one-exciton states have negative effective masses for both branches of Davydov's doublet. However, the dispersion is very small, as one can see in Fig. 4, where the biexciton branch is shown together with free two-exciton bands $(1,1),(1,2)$ and $(2,2)$.

\section{Conclusion}

The presented calculations have shown that gap kinematical biexcitons can exist in crystals with more general structures than considered in the previous paper [6].

\section{Acknowledgements}

We acknowledge the support from the Grant 97-1074 of Russian Ministry of Science and Technology and the Grant 99-03-32178 of Russian Foundation for Basic Research.

\section{References}

[1] V.M. Agranovich, M.D. Galanin, Electronic Excitation Energy Transfer in Condensed Matter, North-Holland, Amsterdam, 1982.

[2] M. Ueta, H. Kanzaki, K. Kobayashi, Y. Toyozawa, H. Hanamura, Excitonic Processes in Solids, Springer, Berlin, 1986.

[3] N.A. Efremov, E.P. Kaminskaya, Sov. Phys. Solid State 15 (1973) 2221.

[4] G. Vektaris, J. Chem. Phys. 101 (1994) 3031

[5] V.M. Agranovich, Theory of Excitons, Nauka, Moscow, 1968.

[6] V.M. Agranovich, O.A. Dubovsky, D.M. Basko, G.C. L Rocca, F. Bassani, J. Luminescence 85 (2000) 221. 